NOTA CIENTÍFICA

\title{
REGISTROS DE PERROS DOMÉSTICOS CON TRAMPAS CÁMARAS EN LA PROVINCIA DE OSORNO, SUR DE CHILE
}

\author{
Fernando García-Solís ${ }^{1}$, Carlos Oyarzún ${ }^{2}$, Jaime R. Rau³ ${ }^{3 *}$ Julio E. Crespo ${ }^{4}$ \\ ${ }^{1}$ Programa de Doctorado en Ciencias, Mención Conservación y Manejo de Recursos Naturales, Chinquihue, \\ Puerto Montt; ${ }^{2} \mathrm{Oficina}$ de Medio Ambiente y Museo de Historia Natural, Purranque, Osorno, Chile; ${ }^{3}$ Laboratorio \\ de Ecología, Departamento de Ciencias Biológicas y Biodiversidad, Universidad de Los Lagos, Osorno, Chile; \\ ${ }^{4}$ Laboratorio de Ciencias Naturales y Sostenibilidad, Departamento de Ciencias Biológicas y Biodiversidad, \\ Universidad de Los Lagos, Osorno, Chile
}

\section{RESUMEN}

Ante los recientes ataques de jaurías de perros sobre ganado bovino, informados en los medios de prensa, se efectuó un monitoreo de perros, para conocer su abundancia en la provincia de Osorno, sur de Chile. Es importante mencionar que los perros pueden transmitir enfermedades infecciosas y otros parásitos como lo son el distemper y parvovirus canino a animales silvestres con los que comparte hábitat. En primavera 2020 y verano 2021 se dispusieron 12 estaciones de muestreo (cada una con una trampa cámara) en la precordillera costera y 13 estaciones similares en la precordillera andina. Durante la primavera 2019 y el verano 2020 se obtuvieron en total 127 fotografías de perros domésticos que correspondieron a 66 animales diferentes, la mayoría de ellos adultos.

Palabras clave: ganado bovino, perros domésticos, sur de Chile, trampas cámaras.

\section{ABSTRACT}

Records of free-roaming dogs with camera traps in the Osorno Province, southern Chile. Before the recent attacks by packs of dogs on bovine cattle reported in the press, their abundance was monitored in the province of Osorno, southern Chile. Moreover, dogs can be vectors of distemper canine virus and parvovirus to wild mammals with which they share the same habitat. In austral spring 2020 and austral summer 2021, sampling stations with 12 camera traps were set up in the coastal foothills and 13 in the Andean foothills. In these seasons 127 overall photographs of domestic dogs were obtained, corresponding to 66 different animals, most of them adults.

Keywords: bovine and ovine cattle, camera traps, domestic dogs, southern Chile.

\section{INTRODUCCIÓN}

En la última década se ha empezado a reconocer el frecuente y elevado impacto negativo de los perros domésticos (Canis lupus familiaris) sobre la abundancia y diversidad de mamíferos nativos en paisajes antropizados (ej. Schüttler et al. 2018, Montecino-Latorre y San Martín 2019, Villatoro et al. 2019, Cortés et al. 2021, Silva-Rochefort y Root-Bernstein 2021). Se ha observado que el impacto de los perros domésticos y asilvestrados sobre la fauna de vertebrados es dependiente del cuidado que presten a ellos sus dueños (Silva-Rodríguez y Sieving 2011). Así, se ha demostrado el efecto depredador de los perros domésticos sobre ciervos endémicos chilenos como el pudú, Pudu puda M. (Silva-Rodríguez et al. 2010) y, en agroecosistemas del sur de Chile, el desplazamiento por competencia de interferencia de los zorros chilla, Lycalopex griseus G. (Silva-Rodríguez et al. 2009). Más recientemente ha comenzado a informarse en los medios de prensa el ataque de jaurías de perros sobre el ganado ovino y bovino. Por otra parte, los perros son transmisores de enfermedades infecciosas y otros parásitos (ej. distemper canino y parvovirus) a mamíferos silvestres con los que comparten hábitat (Acosta-Jamett et al. 2011, Garde et al. 
2013). Debido a ello, el propósito de este estudio es documentar en la provincia de Osorno, sur de Chile, registros fotográficos de perros domésticos con trampas cámaras durante la primavera 2019 y el verano 2020 en la precordillera costera y andina de la provincia de Osorno, sur de Chile. Ellas forman parte de la ecorregión del bosque valdiviano, uno de los 35 "hotspots" de mayor biodiversidad a nivel mundial (Mittermeier et al. 2004).

\section{MATERIAL Y MÉTODOS}

En la provincia de Osorno, sur de Chile, empleando un diseño balanceado se utilizaron trampas cámaras Bushnell (12 en la precordillera costera y 13 en la andina): 6 de 8 MP Trophy Cam HD Hybrid Trail Camera with Nigth Vision, 4 de 24 MP Core Low Glow Trail Camera y 3 de 20 MP Trophy Cam HD Aggressor. Ellas se situaron individualmente en estaciones de muestreo a lo largo de caminos secundarios, puesto que son frecuentados por perros domésticos (Brassine y Parker 2015, Moreira-Arce et al. 2015), enganchadas con cadenas y candados a árboles de gran diámetro a una altura de entre 50-70 cm (Silver et al. 2004), cebadas con atrayente olfativo (Moreira-Arce et al. 2015) y separadas $3 \mathrm{~km}$ unas de otras (Karanth 1995). Diecisiete de las 25 estaciones (68 \%) se instalaron en sitios con un pronunciado efecto antrópico. La disposición de las trampas cámaras se muestra en la Figura 1. Dada la esperada detección diferencial de las especies, los registros fotográficos de éstas se expresaron mediante un índice de densidad relativa (IDR), análogo a los métodos de captura por unidad de esfuerzo (Krebs 1999), el que se obtuvo de dividir el número de registros fotográficos por el esfuerzo de muestreo ( $\mathrm{N}^{\circ}$ de trampas x $\mathrm{N}^{\circ}$ de noches activadas). De esta manera los resultados se entregan como el $\mathrm{N}^{\circ}$ de fotografías/trampa cámara-noche. Para efectos de análisis los registros fotográficos se agruparon en seis categorías: gatos domésticos, aves, mamíferos nativos, mamíferos introducidos, perros domésticos y actividades humanas (caballos, ovejas, terneros, vacas, maquinaria, personas y vehículos). Para comparar los registros fotográficos entre categorías y áreas

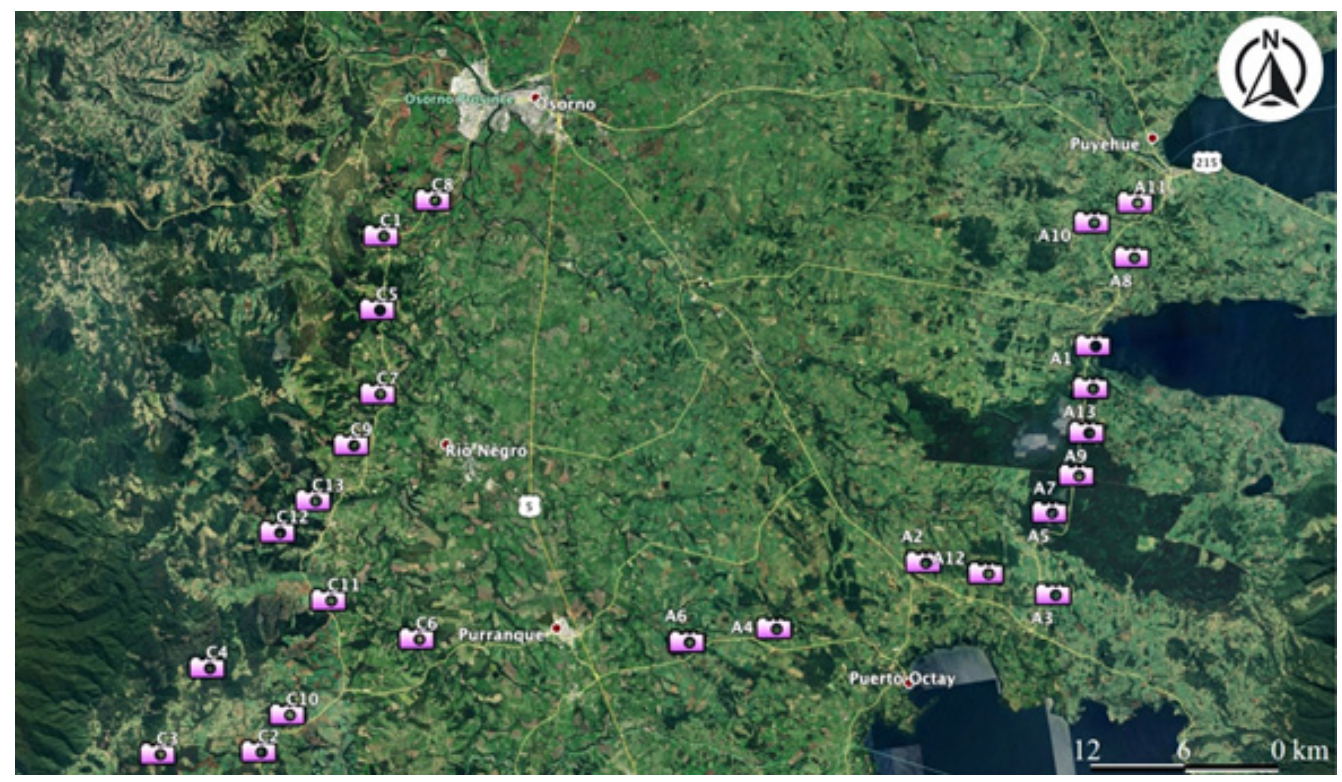

Figura 1. Disposición de trampas cámaras en la precordillera costera y andina de Osorno, sur de Chile. 
se elaboró una tabla de contingencia de 4 filas x 2 columnas (prueba de Chi-cuadrado). Puesto que los datos de abundancia no siguen distribuciones al azar (Brower et al. 1998), para correlacionar los registros fotográficos de perros domésticos con las categorías: a) mamíferos nativos, b) mamíferos introducidos, c) aves y las subcategorías terneros y vacas se usó el coeficiente de correlación no paramétrico de Spearman $\left(\mathrm{r}_{\mathrm{s}}\right)$. Estos análisis estadísticos se realizaron con el paquete computacional en línea VassarStats (http://www. vassarstats.net).

\section{RESULTADOS Y DISCUSIÓN}

Los registros fotográficos de perros domésticos y otras categorías se obtuvieron con trampas cámaras activadas en primavera-verano durante el 31 de noviembre 2019 y el 17 de enero 2020 en la precordillera costera y el 18 de enero 2020 y el 29 de febrero 2020 en la precordillera andina de la provincia de Osorno, sur de Chile. Los resultados se presentan en la Tabla 1. Para 12 estaciones en la precordillera costera se obtuvieron 1.970 fotografías con un esfuerzo de muestreo de 425 trampas-noche (IDR $=4,6$ ) y para 13 estaciones en la precordillera andina 4.401 fotografías con un esfuerzo de muestreo de 441 trampasnoche $(I D R=10,0)$. Para esfuerzos similares los registros fotográficos en la precordillera andina más que duplicaron (2,3 veces) a los de la precordillera costera. En sólo nueve registros no se pudo identificar la categoría fotografiada. Sólo se registraron dos fotografías de gatos domésticos (una en la precordillera costera y otra en la andina). Es sabido que ellos son transmisores de enfermedades infecciosas emergentes a felinos nativos como las guiñas, Leopardus guigna, en la isla de Chiloé, sur de Chile (Mora et al. 2015). Todas las aves fotografiadas fueron especies nativas (bandurrias, Theristicus melanopis; fío-fíos, Elaenia albiceps; picaflores, Sephanoides sephaniodes; queltehues, Vanellus chilensis y traros, Caracara plancus) y de 39 registros fotográficos 29 de ellos correspondieron a la precordillera andina. Para la categoría mamíferos nativos se obtuvieron en la precordillera andina dos fotografías de chingues (Conepatus chinga), tres (dos en la precordillera costera y una en la andina) de guiñas; en la precordillera andina cuatro de pumas (Puma concolor) y 25 fotografías (23 en la precordillera costera y dos en la andina) de zorros chilla. De acuerdo con Fuentes y Jaksic (1979), los zorros chilla usan más los valles que las montañas a diferencia de los zorros culpeos, P. culpaeus. Para la categoría mamíferos introducidos se registraron en la precordillera costera 11 fotografías de ciervos rojos (Cervus elaphus) machos (con cornamentas) y 31 de liebres, Lepus europaeus (18 en la precordillera costera y 13 en la andina). Los ciervos rojos presentan una alta adaptación y capacidad invasora frente a nuevos ambientes (Flueck et al. 2003). En el caso de los perros domésticos se registraron 65 fotografías en la precordillera de la costa y 62 en la andina. En base a diferencias morfológicas de los animales y su tamaño corporal, se estima que el total de fotografías $(\mathrm{N}=$ 127) correspondió a 66 perros domésticos diferentes. De ellos sólo seis $(9,1 \%)$ fueron cachorros.

Comparando las categorías de mamíferos nativos, mamíferos introducidos (ciervos rojos y liebres), perros domésticos y ganado bovino (terneros y vacas) se encontró que las diferencias estuvieron dadas por el mayor registro fotográfico de mamíferos nativos e introducidos en la precordillera costera y de ganado bovino en la precordillera andina (Chi-cuadrado $=884,8 ;$ g.l. $=3 ; \mathrm{P}<0,0001)$. Ninguna correlación de los registros fotográficos de perros domésticos versus mamíferos nativos, mamíferos introducidos, aves, terneros y vacas fue estadísticamente significativa (g.l. $=23 ; \mathrm{P}>0,05)$. En la Cordillera de Nahuelbuta, se ha demostrado que los perros y la actividad humana afectan los patrones de actividad diurna de ocho especies de carnívoros nativos (Moreira-Arce et al. 2015). Los registros fotográficos de actividad humana correspondieron a 1.548 en la precordillera costera y 204 en la precordillera andina, 7,6 veces más en el primer ambiente. Finalmente, en la precordillera costera se registraron 56 fotografías de terneros y 143 de vacas y en la andina sólo cinco fotografías de terneros y 4.072 de vacas. Hubo 28,5 veces más registros de ganado bovino en la precordillera andina que en la costera. Se recomienda monitorear en el área de estudio la abundancia de perros domésticos, en otoño e invierno, para detectar patrones estacionales de abundancia $\mathrm{y}$, anualmente, para conocer tendencias en el tiempo en los cambios de abundancia de estos animales. 


\section{CONCLUSIONES}

Los registros fotográficos de perros domésticos en primavera 2019 y verano 2020 fueron similares, tanto en la precordillera costera como en la precordillera andina de la provincia de Osorno, sur de Chile, dando cuenta de su ubicuidad en el área de estudio. De un total de 127 fotografías, que se estimó que correspondieron a 66 perros, la mayoría de ellos fueron animales adultos. En relación con el ganado bovino, hubo 28 veces más registros fotográficos en la precordillera andina que en la precordillera costera. Por otra parte, la actividad humana fue mayor en la precordillera de la costa comparada con la precordillera andina.

\section{AGRADECIMIENTOS}

Contribución generada por el Área Prioritaria de Investigación Sistema Agroalimentario, Cambio Climático y Biodiversidad (API-3), financiada por la Dirección de Investigación de la Universidad de Los Lagos. Se agradece por su apoyo logístico a la Oficina del Medio Ambiente de la Ilustre Municipalidad de Purranque y a Soraya Sade por la edición del texto y a Ignacio Orellana por la edición de la figura.

\section{REFERENCIAS BIBLIOGRÁFICAS}

ACOSTA-JAMETT, G., W.S.K. CHALMERS, A.A. CUNNIGHAM, S. CLEAVELAND, I.G. HANDEL y B.M. DE C. BRONSVOORT. 2011. Urban domestic dog populations as a source of carnivore distemper virus for wild carnivores in the Coquimbo Región of Chile. Veterinary Microbiology 152: 247-257.

BRASSINE, E. y D. PARKER. 2015. Trapping elusive cats: using intensive camera trapping to estimate density of rare African felids. PloS One 10(12) https://doi.org/10.1371/journal.pone.0142508.

BROWER, J.E., J.H. ZAR y C.N. von ENDE. 1998. Field and laboratory methods for General Ecology. $4^{\text {th }}$ ed. WCB/ McGraw-Hill. Boston, Mass.

CORTÉS, E.I., J.G. NAVEDO y E.A. SILVA-RODRÍGUEZ. 2021. Widespread presence of domestic dogs on sandy beaches of southern Chile. Animals 11(1), 161; https://doi.org/10.3390/ani11010161

FLUECK, W.T., J.A.M. SMITH-FLUECK y C.M. NAUMANN. 2003. The current distribution of red deer (Cervus elaphus) in southern South America. Zeitschrift für Jadwissenchaften 49: 112-119.

FUENTES, E.R. y F.M. JAKSIC. 1979. Latitudinal variation of Chilean foxes: test of alternative hypotheses. Ecology 60: 43-47.

GARDE, E., G. ACOSTA-JAMETT and B.M. BROONSVORT. 2013. Review of the risk of some canine zoonoses from free-roaming dogs in the post-disaster setting of Latin America. Animals 3: 855-865.

KARANTH, K.U. 1995. Estimating tiger Panthera tigris populations from camera-traps data using capture-recapture models. Biological Conservation 71: 331-338.

KREBS, C.J. 1999. Ecological Methodology. 2nd. Ed. Menlo Park, California. 620 pp.

MITTERMEIER, R., N. MYERS y C. GOETTSCH-MITTERMEIER. 2004. Hotspots revisited: earth's biologically richest and most endangered terrestrial ecoregions. Ed. CEMEX, Nature, London.

MONTECINO-LATORRE, D. y W. SAN MARTÍN. 2019. Evidence supporting that human-subsidized free-ranging dogs are the main cause of animal losses in small scale farms in Chile. Ambio 48: 240-250.

MORA, M., C. NAPOLITANO, R. ORTEGA, E. POULIN y J. PIZARRO-LUCERO. 2015. Feline immunodeficiency virus and feline leukemia virus infection in free-ranging guignas (Leopardus guigna) and sympatric domestic cats in human landscapes on Chiloé Island, Chile. Journal of Wildlife Diseases 51: 199-208. 
MOREIRA-ARCE, D., P.M. VERGARA y S. BOUTIN. 2015. Diurnal human activity and introduced species affect occurrence of carnivores in a human-dominated landscape. PloS One; https://doi.org/10.1371/journal.pone.0137854

SCHÜTTLER, L., L. SAAVEDRA-ARACENA y J.E. JIMÉNEZ. 2018. Domestic carnivore interactions with wildlife in the Cape Horn Biosphere Reserve, Chile: husbandry and perceptions of impact from a community perspective. PeerJ 6:e4124; http://dx.doi.org/10.7717/peerj.4124

SILVA-ROCHEFORT, B. y M. ROOT-BERNSTEIN. 2021. History of canids in Chile and impacts on prey adaptations. Ecology and Evolution 11: 9892-9903.

SILVA-RODRÍGUEZ, E.A., G.R. ORTEGA-SOLÍS y J.E. JIMÉNEZ. 2009. Conservation and ecological implications of the use of sace by chilla foxes and free-ranging dogs in a human-dominated landscape in southern Chile. Austral Ecology 35: 765-777.

SILVA-RODRÍGUEZ, E.A., C. VERDUGO, O.A. ALEUY, J.G. SANDERSON, G.R. ORTEGA-SOLÍS, F. OSORIOZÚÑIGA y D. GONZÁLEZ-ACUÑA. 2010. Evaluating mortality sources for the Vulnerable pudu Pudu puda in Chile: implications for the conservation of a threatened deer. Oryx 44: 97-103.

SILVA-RODRÍGUEZ, E.A. y K.E. SIEVING. 2011. Influence of care of domestic carnivores on their predation on vertebrates. Conservation Biology 25: 808-815.

SILVER, S.C, L.E.T OSTRO, L.K. MARSH, L. MAFFEI, A.J. NOSS, M.J. KELLY, R.B. WALLACE, H. GÓMEZ y G. AYALA. 2004. The use of camera traps for estimating jaguar Panthera onca abundance and density using capture/ recapture analysis. Oryx 38: 148-154.

VILLATORO, E.J., L. NAUGTHON-TREVES, M.A. SEPÚLVEDA, P. STOWHAS, F.O. MARDONES y E.A. SILVA-RODRÍGUEZ. 2019. When free-ranging dogs threaten wildlife: public attitudes toward management strategies in southern Chile. Journal of Environmental Management 229: 67-75. 


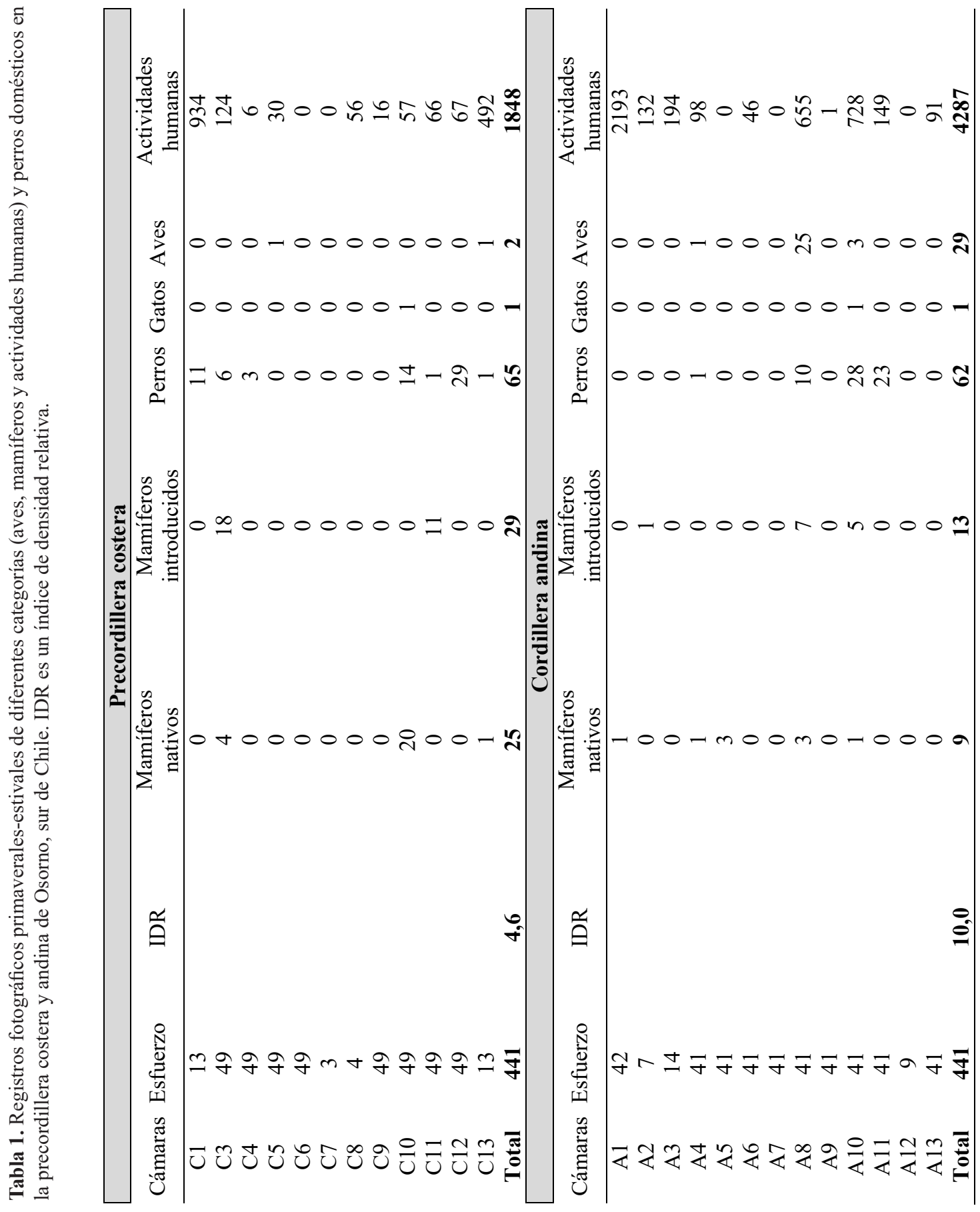

